\title{
A distinct lymphocyte distribution in relapse after rituximab for steroid-dependent nephrotic syndrome
}

\author{
Masaki Shimizu • Tadafumi Yokoyama • \\ Sayaka Ishikawa $\cdot$ Kazuyuki Ueno • \\ Kazuhide Ohta $\cdot$ Akihiro Yachie
}

Received: 11 April 2012/Accepted: 28 June 2012/Published online: 9 August 2012

(C) Japanese Society of Nephrology 2012

\begin{abstract}
Rituximab (RTX) is a new steroid-sparing therapy for childhood steroid-dependent nephrotic syndrome (NS). However, relapses frequently occur immediately after CD19 recovery. We report the cases of two steroid-dependent NS patients treated with RTX followed by mizoribine (MZB). One patient relapsed, and the other developed proteinuria after CD19 recovery until the MZB was replaced by mycophenolate mofetil. These patients exhibited different lymphocyte phenotypes, with the CD4+/CD8+ profile favoring CD8+ T lymphocytes, while CD3+ HLA-DR-expressing activated T lymphocyte expansion occurred in the relapsed patient. Based on these findings, we suggest that $\mathrm{T}$ cell activation may influence outcome and that phenotypic analysis in addition to B cell monitoring may facilitate the detection of NS relapse.
\end{abstract}

Keywords Rituximab - Nephrotic syndrome $\cdot \mathrm{T}$ cell . B cell

\section{Introduction}

Approximately $80 \%$ of children with an initial episode of idiopathic nephrotic syndrome (NS) have minimal change disease and respond to steroid therapy. However, the

\footnotetext{
M. Shimizu $(\bowtie) \cdot T$. Yokoyama - S. Ishikawa · K. Ueno ·

A. Yachie

Department of Pediatrics, School of Medicine, Institute of Medical, Pharmaceutical and Health Sciences, Kanazawa

University, 13-1 Takaramachi, Kanazawa 920-8641, Japan

e-mail: shimizum@staff.kanazawa-u.ac.jp

K. Ohta

Department of Pediatrics, Kanazawa Medical Center,

Kanazawa, Japan
}

remaining $20 \%$ do not respond and are considered to be steroid-resistant [1]. Of the children with steroid-sensitive NS, 50-60\% have frequent relapses of steroid-dependent NS (SDNS) on follow-up. The treatment of children with SDNS and steroid-resistant NS (SRNS) is challenging and often requires treatment with immunosuppressants, such as cyclophosphamide (CPM), cyclosporine A (CsA), tacrolimus, mizoribine (MZR), and mycophenolate mofetil (MMF) [2, 3]. Although useful, treatment with steroids, CsA, or both is associated with significant side effects, such as growth retardation, cataracts, osteoporosis, hypertension, and nephrotoxicity.

The monoclonal anti-CD20 antibody rituximab (RTX) is a novel therapy for childhood SDNS [4-8]. Significant decreases in the frequency of relapse, period of steroid use, and steroid dosages have been documented in patients following RTX administration. However, NS relapse frequently occurs a few months after CD19 recovery [4-8], leading to the proposal that maintenance therapy with an immunosuppressant, such as MMF, is required to prevent relapse after RTX therapy [4].

Although the etiology of idiopathic NS remains obscure, a role for the immune system is strongly suggested. T cells in particular have a major function in NS pathogenesis [9-13]. RTX is effective in treating NS, but the mechanism by which RTX has its effects on NS, the changes that occur in lymphocyte phenotypes during RTX therapy, and the underlying cause of the relapse with B cell recovery after RTX therapy are also still unknown.

Here, we present the cases of two patients with SDNS who were treated with RTX. One patient had a relapse, whereas the other exhibited a transient increase in proteinuria after RTX therapy. We describe the clinical courses of these patients and show the distinct lymphocyte phenotypes during NS relapse with B cell recovery after RTX therapy. 


\section{Case reports}

\section{Case 1}

A Japanese girl was diagnosed with idiopathic NS at age 5 years. Renal biopsy at diagnosis showed minimal change disease. Successful treatment was accomplished with standard prednisolone (PSL) therapy $\left(60 \mathrm{mg} / \mathrm{m}^{2} /\right.$ day, 4 weeks). The first relapse occurred after the PSL dosage had been reduced $\left[60 \mathrm{mg} / \mathrm{m}^{2} /\right.$ alternate day (AD)]. She responded again to steroid treatment, but a second relapse occurred after the PSL dosage had once again been reduced. Treatment with MZR (300 mg/day) was initiated; however, complete remission could not be achieved and the disease subsequently became steroid-dependent. Treatment with CsA was started, but as the patient developed headaches and nausea, CsA was replaced with tacrolimus. However, the patient then developed diarrhea and palpitations. Treatment with low-density lipoprotein apheresis was added to the therapeutic regimen, but the reduction in proteinuria was transient. Despite immunosuppressive therapy with steroids and these immunosuppressive drugs, multiple relapses occurred. We therefore decided to treat the patient with RTX.

Treatment with intravenous infusion of RTX $\left(375 \mathrm{mg} / \mathrm{m}^{2}\right)$ was initiated (Fig. 1). Approval for the off-label use of RTX was obtained from the institutional review board at Kanazawa University, and written informed consent was obtained from the patient's parents. No adverse events were observed. Maintenance therapy with MZR was continued. One week after the initiation of RTX therapy, the B cell count in the peripheral blood dropped to $0 \%$. Within 3 months of RTX therapy, the patient was stable, without NS relapse, and the PSL dosage could be reduced to $10 \mathrm{mg}$ AD. However, 4 months after RTX therapy, NS relapse occurred immediately after B cell recovery. Methylprednisolone pulse therapy ( $0.5 \mathrm{~g} /$ day, every 2 weeks) was initiated; however, significant proteinuria continued. Complete remission was achieved after MZR was replaced with MMF (1,000 mg/day). She has had no relapses for 21 months under the treatment regimen with PSL (30 mg AD) and MMF (1,000 mg/day).

\section{Case 2}

A Japanese boy was diagnosed with idiopathic NS at age 2 years. Treatment with a standard PSL regimen $(60 \mathrm{mg} /$ $\mathrm{m}^{2} /$ day, 4 weeks) was ineffective, and SRNS was diagnosed. Renal biopsy showed focal segmental glomerulosclerosis. Treatment with CPM was started; however, complete remission could not be achieved. The patient responded to CsA, but frequent relapses occurred after the PSL dosage was reduced, and he became steroid- dependent. Treatment with MZR was added to the regimen; however, his steroid dependency remained unchanged. At age 17 years, follow-up renal biopsy showed focal segmental glomerulosclerosis and cyclosporine toxicity. Despite immunosuppressive therapy with steroid and these immunosuppressive drugs, multiple relapses occurred. We therefore decided to treat the patient with RTX.

RTX was intravenously infused $\left(375 \mathrm{mg} / \mathrm{m}^{2}\right)$ once weekly for 2 consecutive weeks (Fig. 2). Approval for the off-label use of RTX was obtained from the institutional review board at Kanazawa University, and written informed consent was obtained from the patient's parents. No adverse events were observed. Maintenance therapy with MZR was continued. One week after the initiation of RTX therapy, B cell count in the peripheral blood dropped to $0 \%$. Within 6 months of RTX therapy, the patient was stable without NS relapse. PSL and CsA dosages were decreased to $10 \mathrm{mg} \mathrm{AD}$ and $120 \mathrm{mg} /$ day, respectively. However, 7 months after RTX therapy, proteinuria increased following CD19 recovery. Complete remission was achieved after the MZR was replaced with MMF (1,500 mg/day). PSL and CsA could be completely discontinued, and he has had no relapses for 18 months under the MMF treatment regimen $(1,500 \mathrm{mg} /$ day $)$.

\section{Alteration in peripheral blood markers during CD19} recovery

In case 1 , the total number of $\mathrm{T}$ cells decreased, and an imbalance in the $\mathrm{CD} 4+/ \mathrm{CD} 8+$ distribution in favor of $\mathrm{CD} 8+\mathrm{T}$ lymphocytes and expansion of CD3+ activated $\mathrm{T}$ lymphocytes expressing HLA-DR was observed during NS relapse after the recovery of CD19+ B cells (Table 1). These findings were consistent with those observed in NS relapse before RTX therapy in this patient, and they improved in remission. In contrast, these changes were not observed in case 2 .

\section{Discussion}

We have presented the case reports of two patients with refractory NS who were treated with RTX. One patient had a relapse, whereas the other exhibited a transient increase in proteinuria following RTX therapy. We have also shown the distinct lymphocyte phenotypes during NS relapse with $\mathrm{B}$ cell recovery after RTX therapy.

The etiology of idiopathic NS remains obscure; however, a role for $\mathrm{T}$ cells with the release of a circulating factor is strongly suggested [9]. There have been many reports recently of the steroid-sparing effect of RTX in NS [4-8], suggesting that not only $\mathrm{T}$ cells but also B cells may play a role in the pathogenesis of NS. The mechanism of RTX 
Fig. 1 Clinical course of case 1. $\mathrm{U}$-Pro/Cr Urine proteinurine creatinine ratio, circles containing numbers/arrows time points at which blood samples were obtained. RTX rituximab, $P S L$ prednisolone, $M Z R$ mizoribine, $M M F$ mycofenolate mofetil, $m P S L$ methylprednisolone

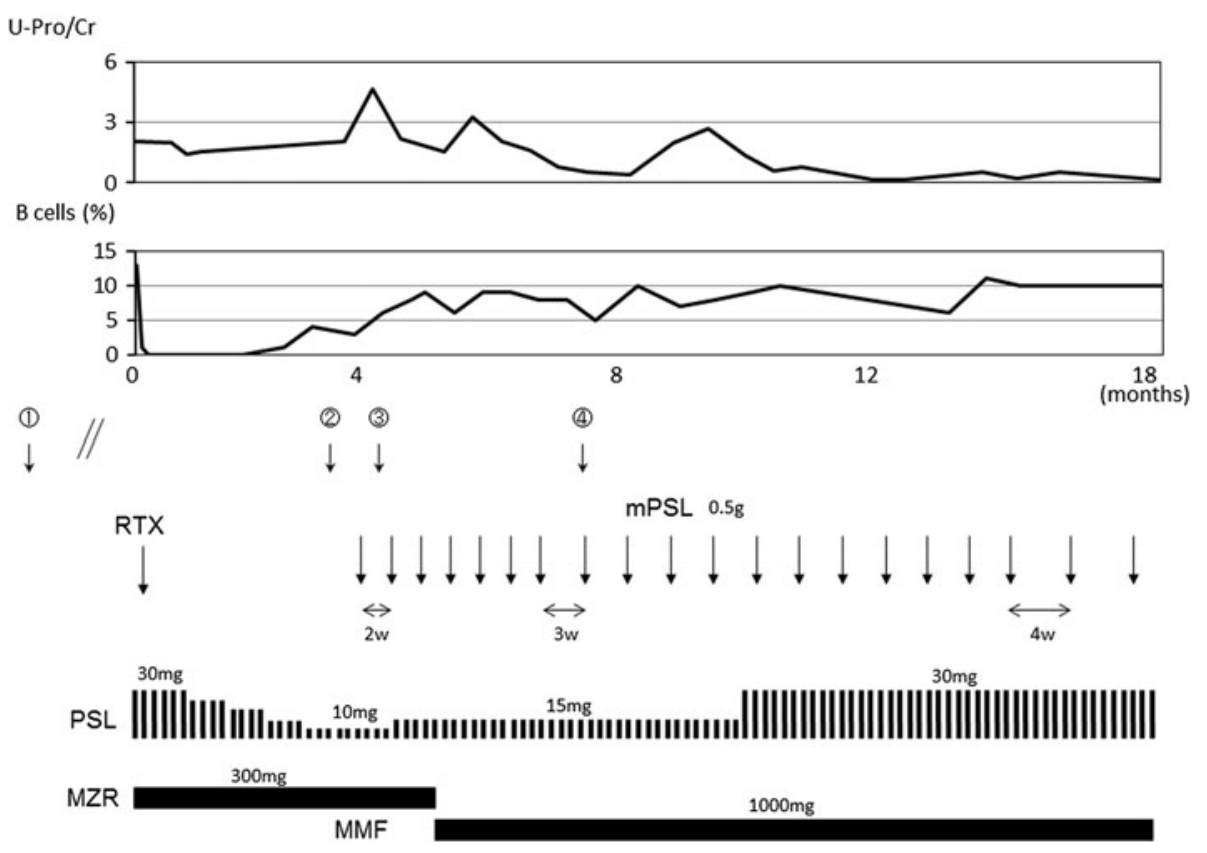

$\mathrm{U}-\mathrm{Pro} / \mathrm{Cr}$

2. CsA cyclosporine

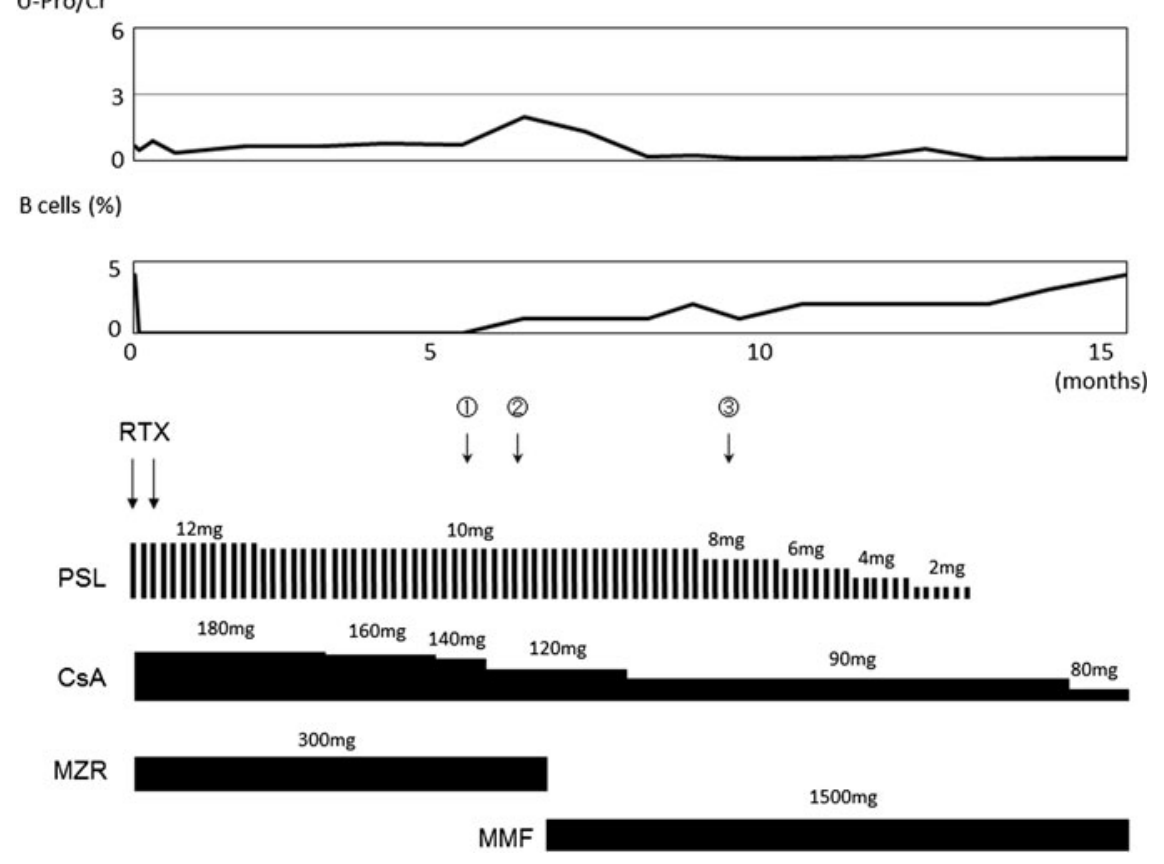

action in NS treatment is also unclear. However, a number of hypotheses have been proposed, including suppression of $\mathrm{T}$ cell activation by the blocking of $\mathrm{T}$ cell-B cell interactions and the induction of regulatory $\mathrm{T}$ cells $[14,15]$.

One important issue related to RTX treatment is that relapses occur frequently a few months after the recovery of B cells. It has been reported that a single infusion of RTX can delete B cells for only about 5 months [4-8]. Thus, the establishment of a proper treatment protocol, such as the number of RTX infusions, timing of the introduction of RTX, and how to taper the PSL dose, is desired. Maintenance therapy is also an option to prevent relapses after RTX treatment. In case 1, maintenance therapy consisted of MZR, and the patient relapsed 4 months after RTX treatment. In case 2, maintenance therapy consisted of CsA and MZR, and while the patient did have increased proteinuria, the switch from MZR to MMF induced complete remission. These findings indicate that MMF might be effective for the maintenance therapy of NS after RTX treatment as Ito et al. [4] reported. 
Table1 Alteration in the level of peripheral blood markers at the phase of CD19 recovery

\begin{tabular}{|c|c|c|c|c|c|}
\hline Cases & $\begin{array}{l}\text { Time points at which } \\
\text { blood was drawn }\end{array}$ & $\mathrm{CD} 3+\mathrm{T}$ cells & CD19+ B cells & $\begin{array}{l}\text { Activated T cells } \\
(\mathrm{CD} 3+\mathrm{DR}+)\end{array}$ & CD4/8 \\
\hline \multicolumn{6}{|l|}{ Case 1} \\
\hline NS relapse before RTX & (1) & $51 \%, 892 / \mu 1$ & $18 \%, 315 / \mu 1$ & $6 \%, 105 / \mu 1$ & 0.82 \\
\hline 2 Weeks before relapse under RTX & (2) & $70 \%, 1,239 / \mu 1$ & $1 \%, 18 / \mu 1$ & $2 \%, 35 / \mu 1$ & 1 \\
\hline Relapse & (3) & $43 \%, 606 / \mu 1$ & $4 \%, 56 / \mu 1$ & $8 \%, 112 / \mu 1$ & 0.56 \\
\hline Remission & (4) & $80 \%, 2,912 / \mu 1$ & $10 \%, 364 / \mu 1$ & $3 \%, 109 / \mu \mathrm{l}$ & 1.53 \\
\hline \multicolumn{6}{|l|}{ Case 2} \\
\hline $\begin{array}{l}3 \text { Weeks before the onset } \\
\text { of proteinuria under RTX }\end{array}$ & (1) & $81 \%, 2341 / \mu 1$ & $1 \%, 29 / \mu 1$ & $4 \%, 116 / \mu 1$ & 1.71 \\
\hline Onset of proteinuria & (2) & $84 \%, 2,780 / \mu 1$ & $1 \%, 33 / \mu l$ & $3 \%, 99 / \mu 1$ & 1.56 \\
\hline Remission & (3) & $81 \%, 1,515 / \mu 1$ & $9 \%, 168 / \mu 1$ & $5 \%, 94 / \mu 1$ & 1.65 \\
\hline
\end{tabular}

$N S$ Nephrotic syndrome, $R T X$ rituximab

${ }^{a}$ See Figs. 1 and 2 for specific information on the time points

The immunosuppressive effects of MMF and MZR result from antipurine metabolite actions. Both of these drugs inhibit inosine monophosphate dehydrogenase (IMPDH), which plays an important role in the de novo synthesis of guanosine nucleotides in $\mathrm{T}$ and $\mathrm{B}$ lymphocytes. MMF is an ester prodrug of the active immunosuppressant mycophenolic acid (MPA). MPA is an uncompetitive and reversible inhibitor of IMPDH, whereas the phosphorylated form of MZB, mizoribine- 5 '-monophosphate, is a competitive inhibitor of IMPDH. One of the differences between MMF and MZB in terms of clinical effectiveness might result from their different mechanisms of action. Another possibility is that the serum concentration of MZR in our patients might not have reached an effective level. High-dose MZB therapy has recently been reported to be effective as a maintenance therapy for SDNS $[16,17]$. Further study is necessary to determine the optimal dose of MZB for patients with SDNS.

Activated $\mathrm{T}$ cell activation might be associated with the relapse of NS [10-13]. A number of studies have revealed an imbalance in the distribution of CD4+ T cells/CD8 + T cells in favor of CD8+ $\mathrm{T}$ cells and the expansion of the CD4+ and $\mathrm{CD} 8+$ memory $\mathrm{T}$ cells that express the CD45RO marker during NS relapse [10-13]. B cell expansion was also recently reported during NS relapses [18]. We previously reported that RTX could suppress $\mathrm{T}$ cell activation in a patient with recurrence of focal segmental glomerulosclerosis after renal transplantation [15]. Interestingly, $\mathrm{T}$ cell activation was observed in our patient (case 1) with NS relapse after the recovery of B cell, whereas this phenomenon was not observed in our patient (case 2) without NS relapse. It is difficult to determine whether $\mathrm{T}$ cell activation is a cause or consequence of NS relapse. However, our results strongly suggest that $\mathrm{T}$ cell activation in cooperation with the recovery of B cell might be associated with NS relapse after RTX treatment. Our results also suggest that RTX can suppress T cell activation in patients with NS by blocking T cell-B cell interactions.

In conclusion, based on our results, we suggest that $\mathrm{T}$ cell activation might play an important role in NS relapse after RTX treatment. The monitoring of not only B cell counts but also phenotypic analysis of T cells may be useful tools by which to detect NS relapse after RTX treatment.

Conflict of interest None.

\section{References}

1. Churg J, Habib R, White RHR. Pathology of nephritic syndrome in children: a report for the International Study of Kidney Disease in Children. Lancet. 1970;760:1299-302.

2. Hodson EM, Alexander SI. Evaluation and management of steroidsensitive nephrotic syndrome. Curr Opin Pediatr. 2008;20:145-50.

3. Moudgil A, Bagga A, Jordan SC. Mycophenolate mofetil therapy in frequently relapsing steroid-dependent and steroid-resistant nephrotic syndrome of childhood: current status and future directions. Pediatr Nephrol. 2005;20:1376-81.

4. Ito S, Kamei K, Ogura M, Sato M, Fujimaru T, Ishikawa T, Udagawa T, Iijima K. Maintenance therapy with mycophenolate mofetil after rituximab in pediatric patients with steroid-dependent nephrotic syndrome. Pediatr Nephrol. 2011;26:1823-8.

5. Fujinaga S, Hirano D, Nishizaki N, Kamei K, Ito S, Ohtomo Y, Shimizu T, Kaneko K. Single infusion of rituximab for persistent steroid-dependent minimal-change nephrotic syndrome after long-term cyclosporine. Pediatr Nephrol. 2010;25:539-44.

6. Guigonis V, Dallocchio A, Baudouin V, Dehennault M, HachonLe Camus C, Afanetti M, Groothoff J, Llanas B, Niaudet P, Nivet H, Raynaud N, Taque S, Ronco P, Bouissou F. Rituximab treatment for severe steroid-or cyclosporine-dependent nephrotic syndrome: a multicentric series of 22 cases. Pediatr Nephrol. 2008;23:1269-79.

7. Sellier-Leclerc AL, Baudouin V, Kwon T, Macher MA, Guérin V, Lapillonne H, Deschênes G, Ulinski T. Rituximab in steroiddependent idiopathic nephrotic syndrome in childhood-follow-up after CD19 recovery. Nephrol Dial Transplant. 2012;27:1083-9. 
8. Takei T, Nitta K. Rituximab and minimal change nephrotic syndrome: therapeutic option. Clin Exp Nephrol. 2011;15:641-7.

9. Shalhoub RJ. Pathogenesis of lipoid nephrosis: a disorder of T-cell function. Lancet. 1974;2:556-60.

10. Lama G, Luongo I, Tirino G, Borriello A, Carangio C, Salsano ME. T-lymphocyte populations and cytokines in childhood nephrotic syndrome. Am J Kidney Dis. 2002;39:958-65.

11. Frank C, Herrmann M, Fernandez S, Dirnecker D, Böswald M, Kolowos W, Ruder H, Haas JP. Dominant T cells in idiopathic nephrotic syndrome of childhood. Kidney Int. 2000;57:510-7.

12. Neuhaus TJ, Shah V, Callard RE, Barratt TM. T-lymphocyte activation in steroid-sensitive nephrotic syndrome in childhood. Nephrol Dial Transplant. 1995;10:1348-52.

13. Yan K, Nakahara K, Awa S, Nishibori Y, Nakajima N, Kataoka S, Maeda M, Watanabe T, Matsushima S, Watanabe N. The increase of memory $\mathrm{T}$ cell subsets in children with idiopathic nephrotic syndrome. Nephron. 1998;79:274-8.

14. Vigna-Perez M, Hernandez-Castro B, Paredes-Saharopulos O, Portales-Pérez D, Baranda L, Abud-Mendoza C, González-
Amaro R. Clinical and immunological effects of rituximab in patients with lupus nephritis refractory to conventional therapy: a pilot study. Arthr Res Ther. 2006;8:R83.

15. Shimizu M, Kitagawa K, Nishio S, Yokoyama T, Furuichi K, Ohta K, Wada T, Yachie A. Successful treatment of recurrent focal segmental glomerulosclerosis after renal transplantation by lymphocytapheresis and rituximab. Transpl Int. 2010;23:e53-5.

16. Honda M. Nephrotic syndrome and mizoribine in children. Pediatr Int. 2002;44:210-6.

17. Fujinaga S, Endo A, Watanabe T, Hirano D, Ohtomo Y, Shimizu T, Kaneko K. Maintenance therapy with single-daily, high-dose mizoribine after cyclophosphamide therapy for prepubertal boys with severe steroid-dependent nephrotic syndrome. Clin Nephrol. 2012 [Epub ahead of print].

18. Kemper MJ, Altrogge H, Ganschow R, Müller-Wiefel DE. Serum levels of immunoglobulins and IgG subclasses in steroid sensitive nephrotic syndrome. Pediatr Nephrol. 2002;17:413-7. 